\title{
Malolactic Fermentation Induced by Lactobacillus plantarum in Malbec Wines from Argentina
}

\author{
M.C. Lerena ${ }^{1,2}$, M.C. Rojo ${ }^{1,2}$, S. Sari ${ }^{1}$, L.A. Mercado ${ }^{1,2}$, S. Krieger-Weber ${ }^{3 *}$, M. Combina ${ }^{1,2}$ \\ (1) Estación Experimental Agropecuaria Mendoza, Instituto Nacional de Tecnología Agropecuaria (INTA), Mendoza, Argentina \\ (2) Consejo Nacional de Investigaciones Científicas y Técnicas (CONICET), Argentina \\ (3) Lallemand, In den Seiten 53, D-70825 Korntal-Münchingen, Germany
}

Submitted for publication: January 2016

Accepted for publication: May 2016

Key words: Lactobacillus plantarum, malolactic fermentation, co-inoculation, malic acid, Malbec

\begin{abstract}
The current market requirements to obtain wines with enhanced complexity and varietal character propose the finding of new microorganisms capable of driving the malolactic fermentation (MLF) and contributing to new and distinctive sensory profiles in wines. During MLF, malic acid naturally present in the must is converted into lactic acid by a decarboxylation reaction driven by lactic acid bacteria. Recently, a new strain of Lactobacillus plantarum suitable for MLF in high-pH wines was selected. This study evaluates the capacity of the L. plantarum V22 starter culture to complete MLF at laboratory and semi-industrial scale in different Malbec musts from Argentina. Malbec musts were co-inoculated with S. cerevisiae D254 and $L$. plantarum V22 to conduct alcoholic fermentation and MLF respectively. Two groups of Malbec musts were identified according to the ability of $L$. plantarum to consume malic acid. A first group, called "High Feasibility for MLF", is characterised by the high viability of $L$. plantarum and the completion of MLF. On the other hand, a second group was identified, termed "Partial Feasibility for MLF", and is characterised by a decreased viability of $L$. plantarum and incomplete MLF. This study shows that the capacity of $L$. plantarum to conduct MLF in Malbec musts is dependent on must composition, further suggesting that the evolution of $\mathrm{pH}$ during the fermentation process, rather than initial $\mathrm{pH}$, is a critical factor affecting the successful completion of MLF.
\end{abstract}

\section{INTRODUCTION}

The transformation of grape must into wine includes complex microbial processes in which yeasts and bacteria conduct alcoholic and malolactic fermentation respectively. Malolactic fermentation (MLF), the enzymatic decarboxylation of L-malic acid to L-lactic acid, is an important step in winemaking that is induced by certain species of lactic acid bacteria (LAB), which are adapted to survive in wine (Constantini et al., 2009). The successful completion of MLF is a critical step for many red wines, and also for some white wines, and has a positive effect on wine quality via a reduction in wine acidity and the sensory modification of wine aroma, as well as the provision of microbial stability (Du Toit et al., 2011). Malolactic fermentation usually takes place in the late stage of alcoholic fermentation, or immediately after the end of it. The successful completion of MLF is dependent on several oenological parameters, such as must/wine $\mathrm{pH}$, alcohol content, temperature and sulphur dioxide $\left(\mathrm{SO}_{2}\right)$ concentration (Lerm et al., 2010).

Lactic acid bacteria in grape juice derive from different sources, such as the grape berry surface, the leaves, stems or winery equipment. Soon after crushing, the LAB population in the grape must generally varies from $10^{3}$ to $10^{4} \mathrm{CFU} /$ $\mathrm{mL}$, which depends largely on the maturity and sanitary conditions of the grapes (Wibowo et al., 1985; RibereauGayon et al., 2006; Du Toit et al., 2011). The main species of LAB present in this stage include Lactobacillus plantarum, L. casei, Leuconostoc mesenteroides and Pediococcus damnosus, as well as Oenococcus oeni in a minor proportion (Lonvaud-Funel, 1995). During alcoholic fermentation, the natural progression of the different LAB species correlates with their sensitivity to increasing ethanol concentrations and resistance to low $\mathrm{pH}$, but for most $\mathrm{LAB}$ growth is limited. After the completion of alcoholic fermentation, yeast activity diminishes and wine LAB are able to grow, leading to the onset of malic acid degradation. However, growing conditions in wine are quite restrictive from a nutritional and metabolic standpoint, and only a few microbial groups are able to resist and successfully induce MLF. Only three genera of LAB are associated with wine, namely Oenococcus (Leuconostoc), Lactobacillus and Pediococcus. Wine $\mathrm{pH}$ is highly selective regarding $\mathrm{LAB}$ growth, and at a $\mathrm{pH}$ below 3.5 only certain strains of $O$. oeni are able to survive and

*Corresponding author: E-mail address: skrieger@lallemand.com

Acknowledgements: Funding for this research was provided by INTA project PNAIyAV 1130032, and by project CAT INTA-Lallemand. M.C. Lerena is a postdoctoral fellow of CONICET 
conduct MLF. Under more favourable conditions, e.g. higher $\mathrm{pH}$, some species of Lactobacillus and Pediococcus are likely to perform MLF (Wibowo et al., 1985; Davis et al., 1988).

As described above, wine LAB that are naturally present in must can perform MLF spontaneously after growing up to a critical population that is necessary to start and achieve malic acid degradation. Spontaneous MLF is the result of different LAB populations growing in the must and depends on the grape sanitary conditions, as well as the physicochemical characteristics of the wine (Costello, 2006). Moreover, the conditions favouring the spontaneous occurrence of MLF imply an easy wine matrix: moderate temperatures and very low or no use of $\mathrm{SO}_{2}$ - thereby increasing the risk of spoilage by potential spoilage microorganisms, which eventually could cause off-odours in the final wines. Control of MLF is crucial for successful winemaking practices. Up to today, several LAB, especially $O$. oeni, have been isolated and are available as dry commercial cultures for direct inoculation in must and wine (Lonvaud-Funel, 1999). Oenococcus oeni is better adapted to the limiting conditions imposed by the wine matrix, and therefore it is the most commonly used selected wine LAB strain under regular winemaking conditions, especially in wines with a $\mathrm{pH}$ of below 3.5. This bacterial species is usually inoculated after alcoholic fermentation (sequential inoculation) (Lonvaud-Funel, 1999), although this practice bears the risk of spoilage by other, undesired microorganisms, which can grow easily already during alcoholic fermentation, especially under conditions of high pH, (Jussier \& De Orduña, 2006; Massera et al., 2009). Coinoculation (i.e. the inoculation of selected wine LAB 24 hours after the inoculation of the selected wine yeast into the must) has not been proposed as an alternative procedure to improve MLF performance (Abrahamse \& Bartowsky, 2012; Bartowsky et al., 2015). It has been postulated that coinoculation would be beneficial to LAB survival, allowing a gradual adaptation to the increasing alcohol concentration, and also because of the better nutritional status of the must in comparison to the harsh conditions at the end of alcoholic fermentation (Krieger-Weber, 2002; Rosi et al., 2003; Fugelsang \& Edwards, 2007).

Although $O$. oeni is certainly the most popular species used to induce MLF in winemaking, the microorganism grows slowly and has high nutritional requirements, causing MLF failure under some restricted conditions. It has been shown that other LAB species are also able to perform MLF and to positively modify wine aroma and flavour (Bartowsky \& Henschke, 1995; Fumi et al., 2010). Indeed, Lactobacillus spp. are found throughout the wine environment, indicating that many strains are adapted to survive under regular winemaking conditions. As a result, Lactobacillus spp. are becoming an interesting alternative as suitable MLF starter cultures, mostly in the search for new attributes that could be beneficial from a sensory standpoint (Du Toit et al., 2011; Bravo-Ferrada et al., 2013). Lactobacillus plantarum has been shown to be the most promising LAB to be used as an inoculum in high-pH wines (Fumi et al., 2010; Du Toit et al., 2011). Moreover, L. plantarum, although classified as a facultative heterofermentor, is homo-fermentative for hexoses, which decreases the risk of acetic acid production and the consequent increase in the volatile acidity of the wine (Lonvaud-Funel, 1999; Ribereau-Gayon et al., 2006). During a selection programme conducted at Sacro Cuore, University of Piacenza, an interesting strain of L. plantarum (V22) capable of inducing MLF in fermenting must and wines was selected (Fumi et al., 2010). Moreover, a higher array of genes coding for enzymes associated with aroma production during MLF has been found in the L. plantarum V22 strain in comparison to various $O$. oeni strains (Mtshali et al., 2010). The current trend of harvesting overripe grapes results in wines with a higher $\mathrm{pH}$, which provides a more favourable environment for the development of native bacteria, and $O$. oeni does not necessarily prevail under these conditions. In Argentina, most of the winegrowing regions have warm climatic conditions, resulting in high-pH wines. Thus, these wines seem to be suitable for the use of L. plantarum as LAB starter culture to conduct MLF. The aim of this work therefore was to assess the feasibility of the strain V22 of L. plantarum to perform MLF in Malbec musts derived from different winegrowing regions of Argentina.

\section{MATERIALS AND METHODS}

\section{Microorganisms and starter culture preparation}

Saccharomyces cerevisiae strain Lalvin ICV D254 was used as a commercial dried preparation, and was kindly provided by Lallemand (Montreal, Canada). Active dried yeast was rehydrated according to the manufacturer's specifications, with the addition of nutrients (Fortiferm, Lallemand, Montreal, Canada), and inoculated into grape musts at a concentration of $300 \mathrm{mg} / \mathrm{L}$.

Lactobacillus plantarum strain V22, provided by Lallemand (Montreal, Canada), was used in freeze-dried form. The bacteria were rehydrated according to the manufacturer's instructions and were inoculated in musts at a concentration of $10 \mathrm{mg} / \mathrm{L} 24 \mathrm{~h}$ after $S$. cerevisiae inoculation (co-inoculation: Lp co). In the experiments performed on a semi-industrial scale, a set of musts was inoculated with O. oeni Lalvin VP41 (Lallemand, Montreal, Canada). Oenococcus oeni Lalvin VP41 was rehydrated following the manufacturer's instructions and was inoculated after alcoholic fermentation - sequential inoculation (Oo sec) - at the same concentration of $10 \mathrm{mg} / \mathrm{L}$.

\section{Grapes and winemaking}

Experiments were performed with Malbec grapes from six different viticultural regions of Argentina: A: 25 de Mayo (San Juan); B: Santa Rosa (Eastern Mendoza); C: Lavalle (Northern Mendoza); D: Lujan de Cuyo (Central-western Mendoza); E: Valle de Uco (Southwestern Mendoza); and F: Alto Valle (Río Negro). Experiments were performed at laboratory scale in food-grade plastic fermenters containing $2 \mathrm{~kg}$ of grape must. Experiments were repeated at a semiindustrial scale (100 L stainless steel tanks) with grapes from two viticultural regions: Lujan de Cuyo (Central-western Mendoza) and Valle de Uco (Southwestern Mendoza). The grapes were picked by hand in March and April 2012 (laboratory-scale experiment), and in March and April 2013 (semi-industrial scale experiment). Grapes were crushed at the INTA experimental winery. Potassium metabisulphite was added (50 ppm) and the must was maintained at room 
temperature for $4 \mathrm{~h}$ before yeast inoculation. Titratable acidity of all the musts was adjusted to a target value of $6.5 \mathrm{~g} / \mathrm{L}$ with food-grade tartaric acid, as described previously (Casassa \& Sari, 2014). For the experiment conducted on laboratory scale, rehydrated yeasts were inoculated in each must batch and, after homogenisation, the musts were fractionated into $2 \mathrm{~kg}$-capacity food-grade plastic fermenters. For the experiments performed on a semi-industrial scale, the musts were fractionated into $100 \mathrm{~L}$ stainless steel tanks and yeast inoculation was performed individually for each experimental unit. Fermentations were done following the standard protocols for Malbec wine vinifications. This included a fermentation/maceration period for seven to 10 days at $25 \pm 2{ }^{\circ} \mathrm{C}$, with the cap being plunged daily. All Malbec wines remained on the skins until the end of MLF in the case of the experiments on the laboratory scale; i.e., 15 days for 25 de Mayo, Santa Rosa, Lavalle and Lujan de Cuyo wines, and 18 days for the wines from Valle de Uco and Alto Valle. In the case of the experiments on a semiindustrial scale, skin contact was maintained until the end of alcoholic fermentation: nine days for Lujan de Cuyo wines and 12 days for wines from Valle de Uco. After the skin contact time, the wines from all treatments (co- and sequential inoculation) were settled, racked and kept in carboys at $22^{\circ} \mathrm{C}$, where they were physically and chemically stabilised with the addition of $\mathrm{SO}_{2} 35 \mathrm{ppm}$. Finally, the wines were chilled to $10^{\circ} \mathrm{C}$ for two weeks, and bottled in $375 \mathrm{~mL}$ dark glass bottles (laboratory scale) and $750 \mathrm{~mL}$ dark glass bottles (semi-industrial scale) without filtration, closed with tin screw caps and stored at $18^{\circ} \pm 2^{\circ} \mathrm{C}$ at the INTA wine cellar. All the experiments were performed in triplicate.

\section{Analytical methods}

Daily monitoring of alcoholic fermentation during the experiments was done by measurement of weight loss and temperature in each individual assay on the laboratory scale. The completion of alcoholic fermentation was estimated when the flasks reached a stable weight for three consecutive days. For the experiments performed on the semi-industrial scale, fermentation monitoring was performed by daily measurement of density and temperature. Alcoholic fermentation was considered complete when a density of $990 \mathrm{mg} / \mathrm{L}$ was reached. Initial and final physicochemical analysis of the wines included measurement of titratable acidity, $\mathrm{pH}$, reducing sugars, Brix degrees, volatile acidity and ethanol, using the standard methods according to the recommendations of the National Institute of Viticulture (INV, 2013). Malolactic fermentation was monitored by the quantification of L-malic acid, using the enzymatic test (Roche, Darmstadt, Germany). In the experiments performed on the semi-industrial scale, the $\mathrm{pH}$ was measured periodically for each experimental unit, using a $\mathrm{pH}$ meter (Altronix TPX1).

\section{Control of LAB viability}

Viable populations of $L$. plantarum strain V22 as well as O. oeni were monitored periodically during both experiments. Different dilutions of the fermentation samples in $0.1 \%$ peptone water adopted to the fermentation stage were seeded into MRS culture medium plates (Britania Labs S.A., Buenos
Aires, Argentina), supplemented with $15 \%$ tomato juice ( $\mathrm{pH}$ adjusted to 4.5 ). The plates were incubated at $28^{\circ} \mathrm{C}$ in an anaerobic atmosphere (GENbag anaer, BioMérieux, Marcy l'Etoile, France) until the appearance of visible colonies. The counts were performed on plates containing from 30 to 300 colonies. In some cases, the different types of colonies were further differentiated through microscopic visualisation after Gram staining. Colonies developing within the first $48 \mathrm{~h}$ showing compatible bacilli cellular morphology, Gram-positive and catalase-negative, were considered as L. plantarum. On the other hand, colonies growing only after five days of incubation, appearing with coccobacilli cellular morphology and chain formation, with Gram-positive and catalase-negative reactions, were considered as $O$. oeni.

\section{RESULTS AND DISCUSSION}

The main objective of this work was to evaluate the capacity of L. plantarum V22 to induce and drive MLF in Malbec musts from the different growing regions of Argentina. To that end, a first experiment on the laboratory scale was conducted in musts with different physicochemical characteristics from six different growing regions (Table 1). As shown in Table 1, the musts had a wide range of different sugar concentrations ( ${ }^{\circ}$ Brix), which allowed us to evaluate the tolerance of L. plantarum to variation in osmotic pressure. $\mathrm{pH}$ was also different for each must, but in all cases $\mathrm{pH}$ values were above $3.6 \mathrm{pH}$ units (Table 1). The malic acid concentration ranged from 0.97 to $2.12 \mathrm{~g} / \mathrm{L}$ (Table 1). Alcoholic fermentation in all musts showed regular kinetics, with the duration ranging from 12 to 16 days, depending on the initial sugar concentration of the must (data not shown). Initial counts for L. plantarum post-inoculation were over $10^{6} \mathrm{CFU} / \mathrm{mL}$ in all cases, even in those in which the sugar concentration was high (Fig. 1), suggesting a very good osmotic tolerance of the bacteria. Bacterial counts remained elevated during the first days of active fermentation (five to seven days), showing viable populations over $10^{4} \mathrm{CFU} / \mathrm{mL}$ for all musts; nonetheless, malic acid degradation was not observed during this period (Fig. 1). After this initial period, both L. plantarum viability and malic acid degradation showed two distinct patterns of behaviour: partial and not partial feasibility for MLF. Both groups were arbitrarily defined according to the viability of L. plantarum along the assay and its ability to consume the malic acid present in the must. Thus, the first group of musts was termed "High feasibility for MLF", and in these musts, L. plantarum was able to metabolise $100 \%$ of the initial malic acid present in the must. The "High feasibility for MLF" group included the winegrowing regions of 25 de Mayo (Fig. 1A), Santa Rosa (Fig. 1B) and Lavalle (Fig. 1C). In this group, despite the bacterial population showing a gradual decrease, residual bacteria viability was conserved until the end of the malolactic fermentation (Fig. 1A, 1B, 1C).

The second group of musts was termed "Partial feasibility for MLF". In these musts, L. plantarum was only able to partially degrade the malic acid present in the must. Between 24 and $57 \%$ of malic acid was consumed by the detectable populations of L. plantarum. The "Partial feasibility for MLF" group included must from the winegrowing regions of Lujan de Cuyo (Fig. 1D), Valle de Uco (Fig. 1E) and Alto 


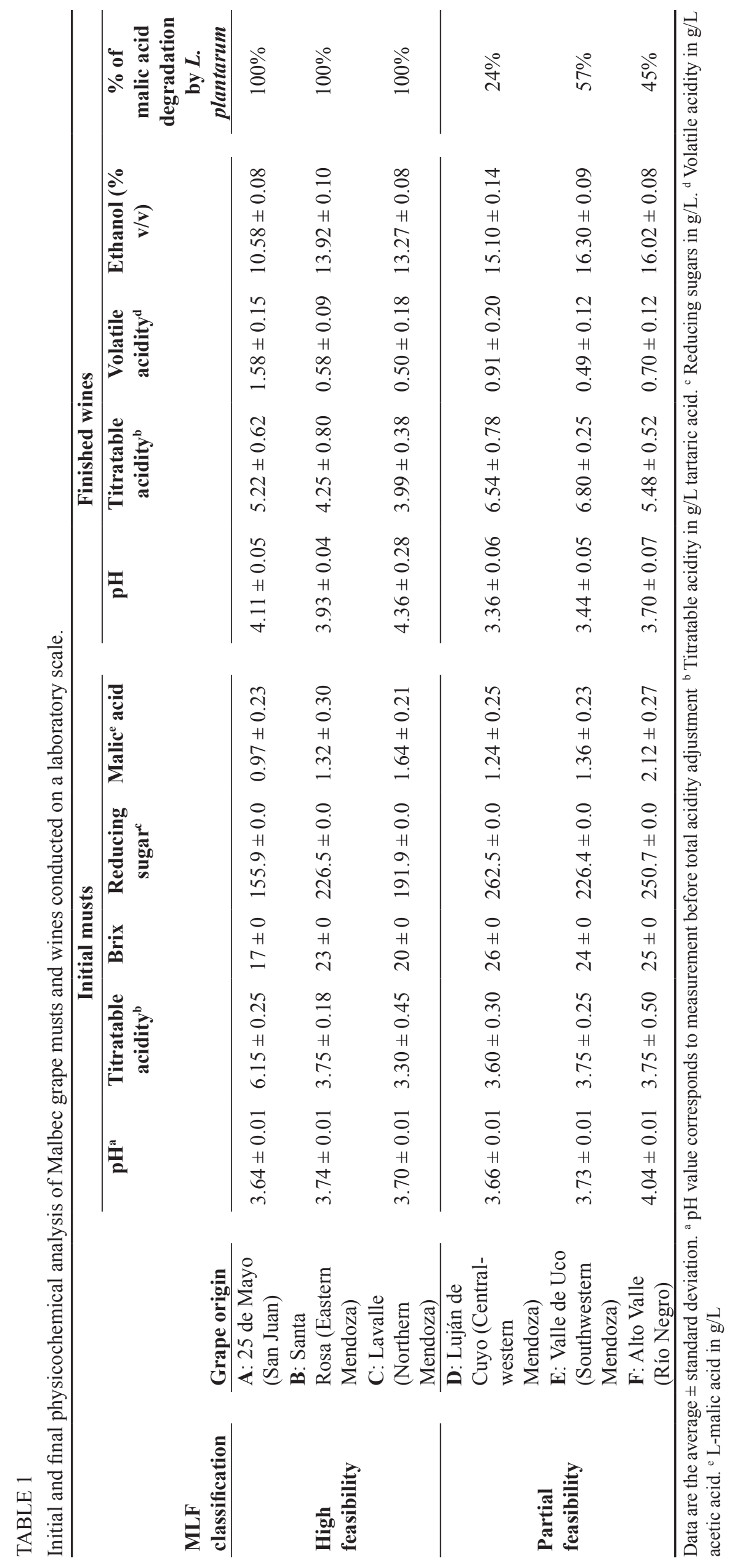


Valle (Fig. 1F). This group showed very good survival of L. plantarum post-inoculation, and the viability generally was maintained during the first days of alcoholic fermentation. However, a sharp drop in bacterial viability was observed in the wines deriving from these three regions from day 6 to day 12 of fermentation (Fig. 1E, 1F, 1G). By day 9 of 12 of fermentation, no viable L. plantarum cells could be detected. Nonetheless, from day 7 (Lujan de Cuyo) and

\section{HIGH FEASIBLITY FOR MLF}

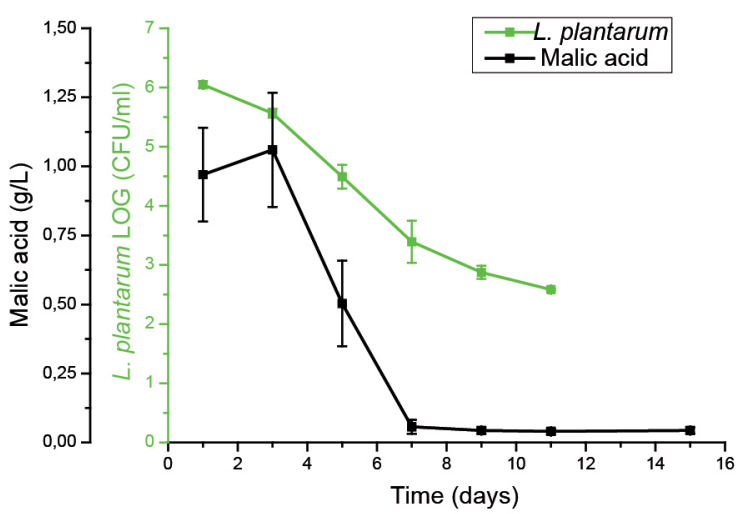

A- 25 de Mayo (San Juan)

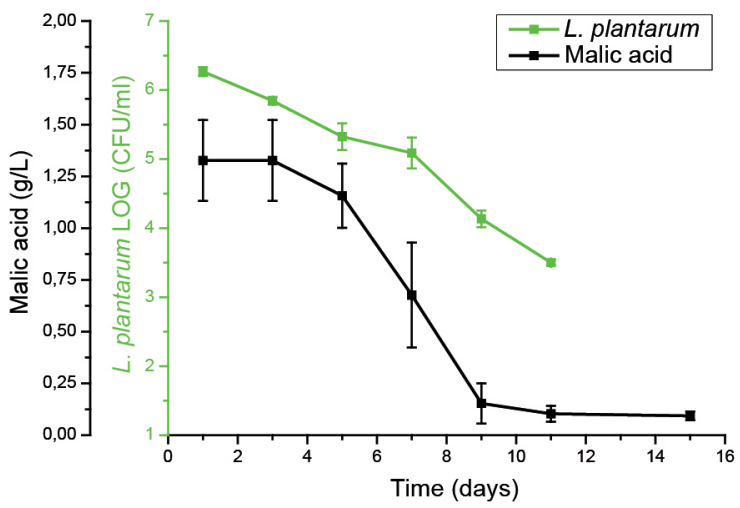

B- Santa Rosa (Mendoza Eastern)

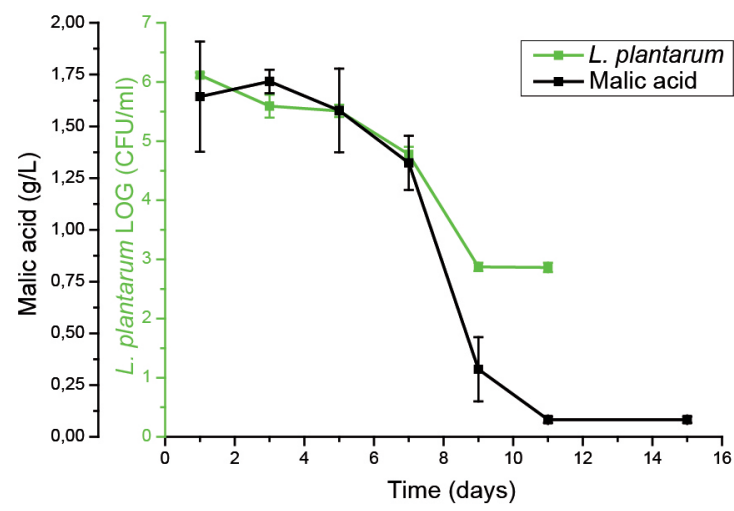

C- Lavalle (Mendoza Northern)

\section{PARTIAL FEASIBLITY FOR MLF}

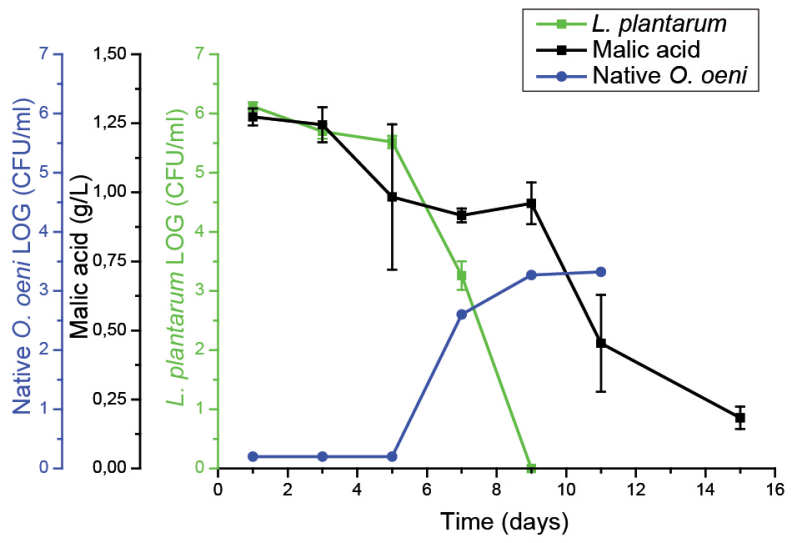

D- Lujan de Cuyo (Mendoza Centerwestern)

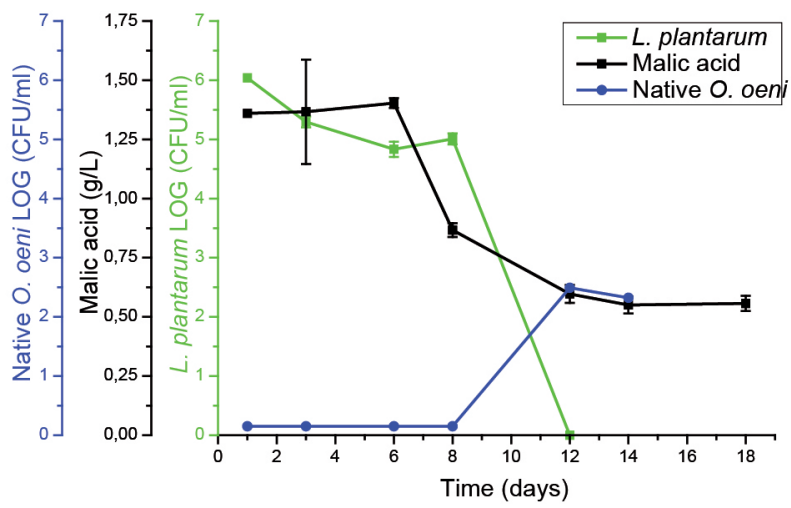

E- Valle de Uco (Mendoza Southwestern)

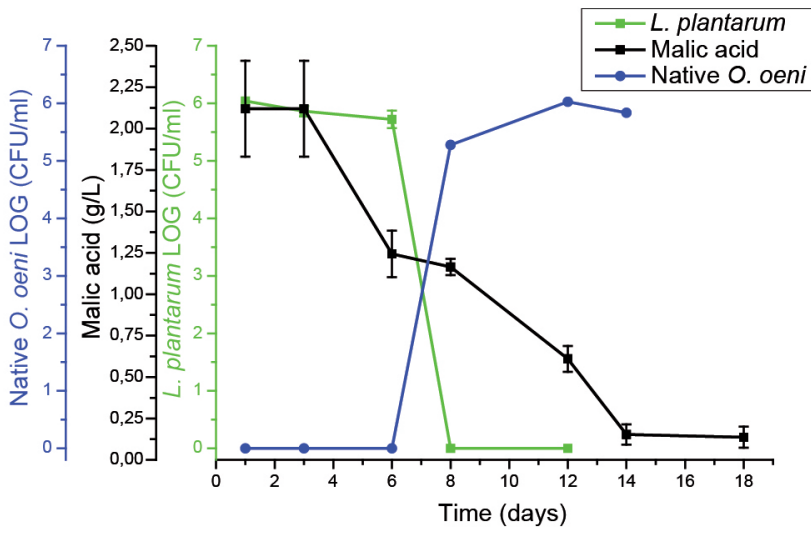

F- Alto Valle (Rio Negro)

FIGURE 1

Kinetics of MLF in vinifications on a laboratory scale using Malbec musts from different regions of Argentina: (A) 25 de Mayo (San Juan), (B) Santa Rosa (Eastern Mendoza), (C) Lavalle (Northern Mendoza), (D) Lujan de Cuyo (Central-western Mendoza), (E) Valle de Uco (Southwestern Mendoza) and (F) Alto Valle (Río Negro). Two groups of regions were identified according to the kinetics of MLF: "High feasibility for MLF" (left) and "Partial feasibility for MLF" (right). Measurements of malic acid concentration $(\mathrm{g} / \mathrm{L})$ (black square), L. plantarum viable cell counts (LOG CFU/mL) (green square) and native $O$. oeni viable cell counts (LOG CFU/mL) (blue circles). 
day 12 (Valle de Uco and Alto Valle), an increasing number of colonies comparable to $O$. oeni bacteria were observed on the plates. As mentioned above, in the group "partial feasibility for MLF", malic acid degradation that could be attributed to L. plantarum metabolism never surpassed $57 \%$. Further consumption of residual malic acid observed in these wines could be related to the metabolism of native $O$. oen $i$ developing afterwards. Consequently, complete malic acid degradation could also be observed in the wines of Lujan de Cuyo and Alto Valle (Fig. 1D and 1F).

The physicochemical composition of the group of "High feasibility for MLF" was characterised by musts with a sugar concentration of below $23^{\circ} \mathrm{Brix}$, and consequently the ethanol content of the resulting wines was not higher than $13.9 \%$ (Table 1). Also, a common feature of the musts from this group was that the final $\mathrm{pH}$ of the wines was significantly higher than the initial $\mathrm{pH}$ of the must. This increase in $\mathrm{pH}$ could have favoured L. plantarum survival in the wines in this group (Table 1). In contrast, the physicochemical properties of the musts of the group with "Partial feasibility for MLF" revealed harsher conditions for L. plantarum survival. As shown in Table 1, all must from this group had fairly high initial sugar levels (between 24 and $26.1^{\circ}$ Brix), and consequently the wines obtained had alcohol levels in the range of 15.1 to $16.3 \%$. Also, the evolution of $\mathrm{pH}$ in this group contrasted with the trends observed in the first group, showing final wine $\mathrm{pH}$ lower than the initial $\mathrm{pH}$ of the must (Table 1). It is important to highlight that changes in the $\mathrm{pH}$ values of Malbec wines from Argentina (after AF and before MLF) have not been reported. Nevertheless, some studies have evaluated the physicochemical characteristics of Malbec wines from different regions of Mendoza, finding average $\mathrm{pH}$ values after MLF of $3.76 \pm 1.08$ (Fanzone et al., 2010; King et al., 2014).

In a subsequent experiment, wines from two regions were re-evaluated on a semi-industrial scale using grapes from Lujan de Cuyo (Central-western Mendoza) and Valle de Uco (Southwestern Mendoza). In this experiment, the fermentations were conducted at a scale that allowed the reproduction of industrial winemaking. Lactobacillus plantarum was inoculated $24 \mathrm{~h}$ after inoculation with S. cerevisiae (co-inoculation: Lp co). A treatment with sequential inoculation of $O$. oeni after alcoholic fermentation (Oo sec) was included in order to compare MLF efficiency to the most frequent procedure used for controlled MLF in the wine industry. In coherence with previous observations in the laboratory-scale experiments, no change in the development of alcoholic fermentation due to the early inoculation with L. plantarum was observed when comparing with the reference treatment with $O$. oeni (Oo sec) (data not shown). In Lujan de Cuyo and Valle de Uco musts, alcoholic fermentation finished within eight to 10 days. Looking at the survival of L. plantarum post-inoculation in both musts, populations over $10^{6} \mathrm{CFU} / \mathrm{mL}$ could be recovered, again showing a good initial adaptation of $L$. plantarum to the high initial osmotic pressure in these musts (Fig. 2A and 2C). In both musts, a gradual decrease of $L$. plantarum viability was observed as the alcoholic fermentation progressed, similar to previous observations in the experiments conducted on a laboratory scale (Fig. 1). Nevertheless, in the Lujan de
Cuyo musts, the drop in viable cells of $L$. plantarum was not as severe as the mortality observed in the Valle de Uco musts, and the final populations of L. plantarum remained over $10^{3} \mathrm{CFU} / \mathrm{mL}$, which allowed the finishing of MLF in the must from Lujan de Cuyo in 26 days (Fig. 2A). In contrast, a steady loss in L. plantarum viability was observed in the Valle de Uco musts, and by day 24 of the fermentation, no more viable cells could be recovered (Fig. 2C). Notably, from day 24 , colonies comparable with native $O$. oeni appeared on the plates (Fig. 2C). The experiment was stopped at day 38 of the fermentation, leaving final concentrations of malic acid of $0.86 \mathrm{~g} / \mathrm{L}$ in the Valle de Uco wines. In the musts from Lujan de Cuyo and Valle de Uco inoculated with $O$. oeni, $100 \%$ of malic acid was degraded (Table 2). Regarding musts inoculated with L. plantarum, in the case of Lujan de Cuyo, $100 \%$ of malic acid was degraded, whereas in the must form Valle de Uco only 48\% degradation was achieved with L. plantarum (Table 2). With regard to treatments sequentially inoculated with $O$. oeni, a good initial survival of $O$. oeni was observed, as well as a steady increase in the populations until the end of the experiment (Fig. 2B and 2D). O. oeni was able to consume $100 \%$ of the malic acid present in the must within 17 to 23 days post-inoculation (Fig. 2B and 2D).

In the semi-industrial scale experiment, the evolution of the $\mathrm{pH}$ along the entire fermentation process also was followed (Fig. 2A to 2D). Surprisingly, we observed, in the case of the Lujan de Cuyo modality, that $\mathrm{pH}$ first dropped after titratable acidity correction, followed by a continuous increase over time, reaching a final wine $\mathrm{pH}$ higher than the initial one in the must (Fig. 2A and Table 2). This $\mathrm{pH}$ behaviour was related to L. plantarum survival, as well as malic acid degradation and, ultimately, to the success of MLF. In contrast, $\mathrm{pH}$ evolution in the Valle de Uco musts showed the opposite behaviour, as $\mathrm{pH}$ initially decreased after titratable acidity correction, followed by a further continuous and steady decrease along the alcoholic fermentation progress (Fig. 2C and Table 2). In this case, the final $\mathrm{pH}$ of the wines was considerably lower than the initial must $\mathrm{pH}$, and consequently L. plantarum viability decreased with time, as did the speed of malic acid consumption (Fig. 2C). These results, together with the results obtained on the laboratory scale (Fig. 1), suggest that L. plantarum survival, as well as its capacity for degrading malic acid in musts, is strongly dependent on the evolution of $\mathrm{pH}$ during alcoholic fermentation, rather than on the initial must $\mathrm{pH}$ or Brix. Very differently, O. oeni showed similar viability in the wines from both regions, regardless of the $\mathrm{pH}$ value at the moment of inoculation, confirming that this microorganism is not sensitive to low $\mathrm{pH}$. However, malic acid degradation was slower in the Valle de Uco wines than the Lujan de Cuyo wines, suggesting that low $\mathrm{pH}$ may not affect bacterial viability in the case of $O$. oeni, but that it still could have a negative effect on the development of malolactic fermentation. Also, it is possible that other stresscausing factors besides $\mathrm{pH}$ value present in the wine from Valle de Uco could affect malic acid degradation (Fig. 2B and 2D).

In recent years, research in the oenological field has focused on different ways to improve the MLF process, as well as on the development of new strains that are able 


\section{LUJAN DE CUYO (MENDOZA CENTER)}

A

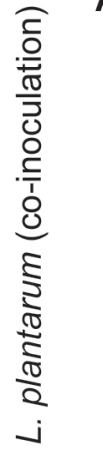

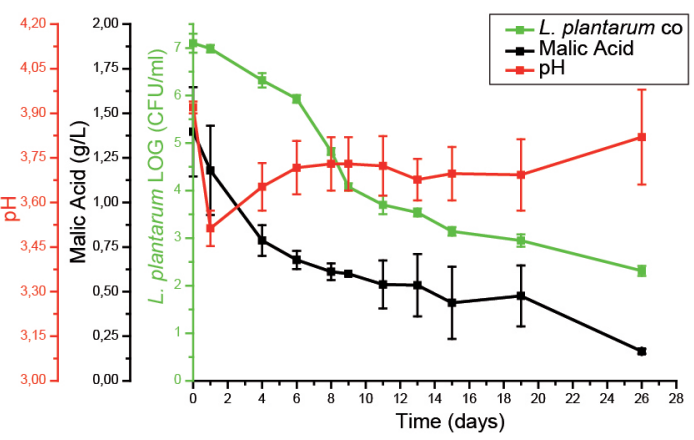

$B$

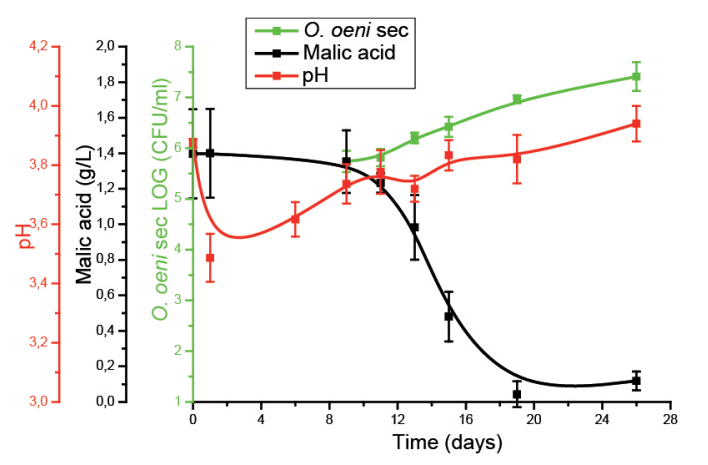

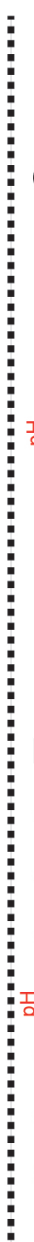

VALLE DE UCO

(MENDOZA CENTER-SOUTH)

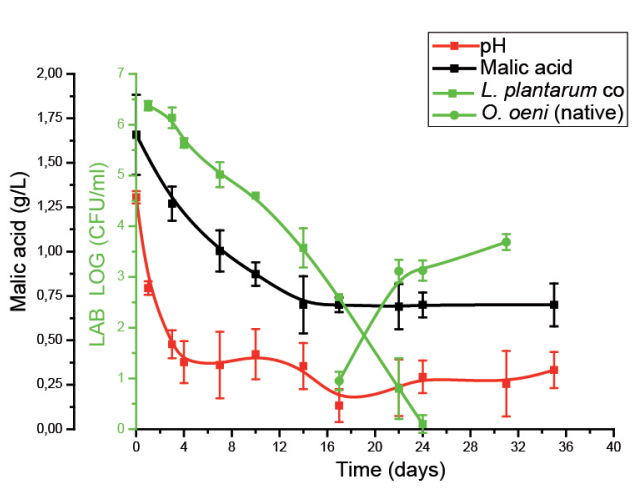

D

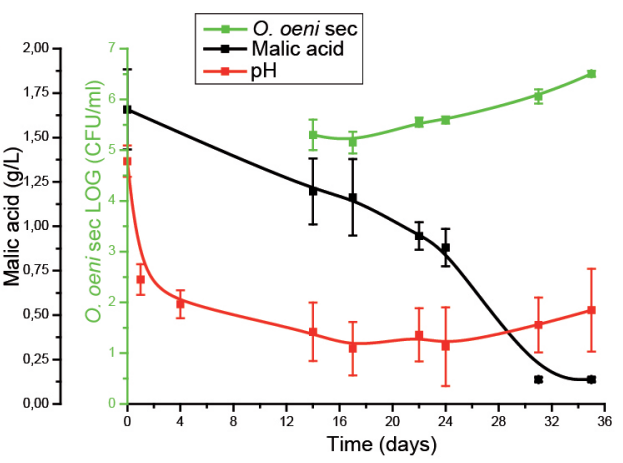

FIGURE 2

Kinetics of MLF in vinifications on a semi-industrial scale using Malbec musts from Lujan de Cuyo (left) and Valle de Uco (right). L. plantarum was inoculated following a co-inoculation procedure in musts from Lujan de Cuyo (A) and from Valle de Uco (C). Another set of wines received sequential inoculation using O. oeni (Lujan de Cuyo (B) and Valle de Uco (D)). Monitoring of malic acid concentration (g/L) (black), LAB viable cell counts (LOG CFU/mL) (green), and $\mathrm{pH}$ (red) was done through the entire fermentation process.

to successfully metabolise the malic acid present in the wine. In this context, we studied the feasibility of using the L. plantarum V22 strain as a ML starter culture in Malbec wines from Argentina. In our experiments we inoculated L. plantarum at the beginning of the alcoholic fermentation, the so-called co-inoculation procedure (inoculation of selected wine LAB 24 hours after inoculation with selected wine yeast). Inoculation in the different musts was successful, as a good initial survival of the bacteria was observed upon inoculation, suggesting a good osmotolerance for L. plantarum. In both sets of experiments, the laboratory scale and semi-industrial scale, a good initial survival of inoculated $L$. plantarum culture was observed, with viable cell counts over 1 x $10^{6} \mathrm{CFU} / \mathrm{mL}$. In line with Abrahamse and Bartowsky (2012), we did not observe any effect of the inoculated bacteria on the rate and evolution of alcoholic fermentation, suggesting no negative interaction between the selected wine LAB and the inoculated wine yeasts.

From the results obtained on the laboratory scale we identified two different Malbec must groups, termed as "High feasibility for MLF" and "Partial feasibility for MLF".
In the second group, malic acid consumption was incomplete (below 57\% - fermentations were monitored up to 35 days). In contrast, Fumi et al. (2012) have reported complete malic acid consumption by L plantarum occurring after almost 50 days in Pinot Noir wines following sequential inoculation.

In both of the experiments, no increase in L. plantarum population was ever observed. In contrast, we observed a decrease in viability occurring with different intensities in both experiments - on the laboratory scale, as well as in the experiments performed on the semi-industrial scale. Nevertheless, even when bacterial viability decreased with time, the remaining bacterial enzymatic activity was able to complete MLF in the musts from 25 de Mayo (San Juan), Santa Rosa (Eastern Mendoza) and Lavalle (Northern Mendoza), all belonging to the "High feasibility for MLF" group, and also in the must from Lujan de Cuyo, in the experiment performed on a semi-industrial scale. These observations are in line with other studies, such as that by Bravo-Ferrada et al. (2013), who followed MLF in Pinot Noir wines inoculated with L. plantarum. Also, in a study by Cho et al. (2011), in which MLF was induced in Grauburgunder 


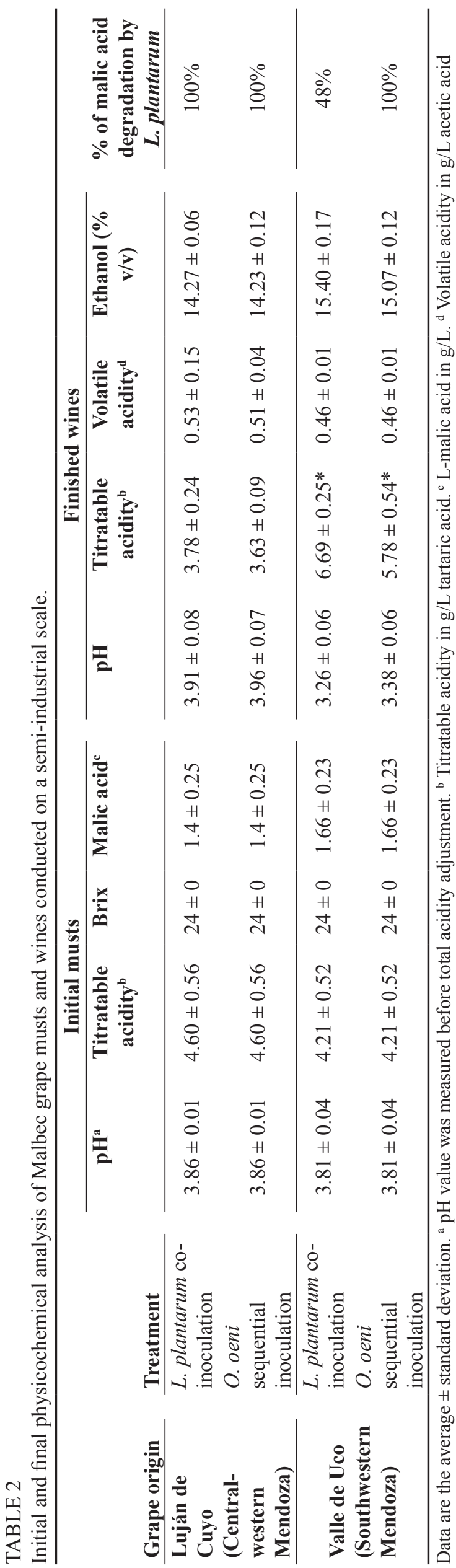

white wine by a strain of $L$. plantarum, a steady decrease in cell counts again was observed in all the fermentations. The kinetics of survival were very different from that observed for $O$. oeni, where we could observe a steady increase in cell counts upon inoculation, along with fast and total malic acid consumption (Fig. 2B and 2D). Nevertheless, in this study we demonstrated that L. plantarum is able to either totally or partially consume malic acid, despite the observed decrease in bacterial survival. This finding allows us to hypothesise that, even when the bacteria are not able to increase their population after inoculation in the fermented grape must, they can still successfully conduct MLF.

Another interesting observation was related to the $\mathrm{pH}$ effect on bacterial survival, and hence on MLF completion. It has been demonstrated that $\mathrm{pH}$ has a selective effect on the LAB species responsible for conducting MLF. $\mathrm{pH}$ values below 3.5 support only $O$. oeni survival and the metabolism of malic acid (Vaillant et al., 1995; Du Toit et al., 2011). Previous studies have demonstrated that L. plantarum is particularly sensitive to low $\mathrm{pH}$, although some strains can even tolerate high ethanol concentrations and consume malic acid, but then the $\mathrm{pH}$ has been close to or above $3.8 \mathrm{pH}$ units (Miller et al., 2011). The same authors established optimum conditions in terms of $\mathrm{pH}$ and ethanol content to assure cell viability and membrane integrity in order to enable oenological L. plantarum strains to consume malic acid in synthetic wine media. However, $\mathrm{pH}$ evolution during the time course of MLF was not monitored in the mentioned study. In our experiments we observed that bacterial survival was related more to $\mathrm{pH}$ evolution during MLF, rather than to the initial $\mathrm{pH}$ of the must. In the experiments conducted on the laboratory scale, as well as those conducted on a semiindustrial scale, Malbec musts showed initial $\mathrm{pH}$ values over 3.6 in all cases (Tables 1 and 2), suggesting suitable conditions for L. plantarum inoculation. However, the $\mathrm{pH}$ showed dynamic behaviour, changing as MLF progressed. More precisely, when $\mathrm{pH}$ decreased over time, bacterial counts declined accordingly, as did the rate of malic acid consumption. This occurred even with initial $\mathrm{pH}$ values of over $3.6 \mathrm{pH}$ units. In contrast, in those fermentations in which $\mathrm{pH}$ increased over the time, L. plantarum was able to successfully metabolise almost all the malic acid originally present in the must, even though a decrease in bacterial survival was observed. Regarding this observation of changes in must/wine $\mathrm{pH}$ and their impact on the malolactic fermentation kinetics of $L$. plantarum, there is a void of scientific literature on $\mathrm{pH}$ evolution during alcoholic and $\mathrm{ML}$ fermentation; most reports are limited to the measurements of initial and final must/wine $\mathrm{pH}$. Our results, on the other hand, suggest that the kinetics of wine $\mathrm{pH}$ during the course of wine fermentation are quite unpredictable and depend much on the buffer capacity of the particular must employed. The latter observation is supported by the fact that, in our studies conducted on the laboratory scale, must composition was highly variable, as the musts were sourced from different Argentinian growing regions. Indeed, must composition is much more complex and unpredictable than that of the usual synthetic wine media used in other studies (Miller et al., 2011). Nevertheless, pH can also be influenced by yeast metabolism, as well as by the release of components 
from the grapes over the course of fermentation. Therefore, more research is needed in order to accurately understand $\mathrm{pH}$ changes during fermentation, their effect on bacterial survival, and the behaviour of the latter as a MLF starter

\section{CONCLUSIONS}

This study supports the importance of Lactobacillus for the new generation of MLF culture starters, not only as an efficient microorganism in terms of malic acid degradation, but also as a promising microbiological tool for early inoculation. Further studies are needed on the specific sensory contribution of $L$. plantarum to the wine aroma profile.

\section{LITERATURE CITED}

Abrahamse, C.E. \& Bartowsky, E.J. 2012. Timing of malolactic fermentation inoculation in Shiraz grape must and wine: Influence on chemical composition. World J. Microb. Biot. 28(1), 255-265.

Bartowsky, E.J. \& Henschke, P.A., 1995. Malolactic fermentation and wine flavor. The Aust. Grapegrower \& Winemaker, Annual Technical Issue, 8394.

Bartowsky, E.J., Costello, P.J. \& Chambers, P.J. 2015 Emerging trends in the application of malolactic fermentation. Aust. J. Grape Wine Res. 21, 663-669.

Bravo-Ferrada, B.M., Hollmann, A., Delfederico, L., Valdés La Hens, D., Caballero, A. \& Semorile, L., 2013. Patagonian red wines: Selection of Lactobacillus plantarum isolates as potential starter cultures for malolactic fermentation. World J. Microb. Biot. 29(9), 1537-1549.

Casassa, L.F. \& Sari, S.E., 2014. Sensory and chemical effects of two alternatives of prefermentative cold soak in Malbec wines during winemaking and bottle ageing. Int. J. Food Sci. Tech. 50(4), 1044-1055.

Cho, G.S., Krauss, S., Huch, M., Du Toit, M. \& Franz, M.P., 2011. Development of a quantitative PCR for detection of Lactobacillus plantarum starters during wine malolactic fermentation. J. Microbiol. Biotech. 21(12), 1280-1286.

Constantini, A., García-Moruno, E. \& Moreno-Arribas, M.V., 2009. Biochemical transformations produced by malolactic fermentation. In: Moreno Arribas, M.V. \& Polo, M.C. (eds.). Wine chemistry and biochemistry. Springer Science and Business Media, New York, USA. pp. 27-57.

Costello, P., 2006. The chemistry of malolactic fermentation. In: Morenzoni, R. (ed.). Malolactic fermentation in wine - Understanding the science and the practice. Lallemand, Montreal. pp. 1-11.

Davis, C., Wibowo, D., Fleet, G. \& Lee, T., 1988. Properties of wine lactic acid bacteria: Their potential enological significance. Am. J. Enol. Vitic. $39(2), 137-142$

Du Toit, M., Engelbrecht, L., Lerm, E. \& Krieger-Weber, S., 2011. Lactobacillus: The next generation of malolactic fermentation starter cultures - An overview. Food Bioprocess Tech. 4(6), 876-906.

Fanzone, M., Peña-Neira, A., Jofre, V., Assof, M. \& Zamora, F., 2010. Phenolic characterization of Malbec wines from Mendoza Province (Argentina). J. Agric. Food Chem. 58, 2388-2397.
Fugelsang, K. C. \& Edwards, C. G., 2007. Fermentation and postfermentation processing. In: K.C. Fugelsang \& C.G. Edwards (eds). Wine Microbiology (2nd edition): Practical Applications and Procedures. Springer, New York. pp. 115-138.

Fumi, M.D., Krieger-Weber, S., Deléris-Bou, M., Silva, A. \& Du Toit, M., 2010. A new generation of malolactic starter cultures for high $\mathrm{pH}$ wines. Proc. Intern. IVIF Congress. IVIF Congress March 2010 Stuttgart.

INV (Instituto Nacional de Vitivinicultura)., 2013. Argentinean Government. Resolución mayo de 1938 - Dirección Nacional de Química. Available at: http://www.inv.gov.ar/index.php/fis-vit-normativas (accessed 9 December 2013).

Jussier D. \& De Orduña, M., 2006. Effect of simultaneous inoculation with yeast and bacteria on fermentation kinetics and key wine parameters of cool-climate Chardonnay. Appl. Environ. Microbiol. 72(1), 221-227.

King, E.S., Stoumen, M., Buscema, F., Hjelmeland, A.K., Ebeler, S.E., Hildegarde, H. \& Boulton, R.B., 2014. Regional sensory and chemical characteristics of Malbec wines from Mendoza and California. Food Chem. 143: 256-267.

Krieger-Weber, S., 2002. Starter cultures for the malolactic fermentation - Time of inoculation. Proc. 13th Intern. Enol. Symp., June 2002, Breisac, Germany. pp. $77-91$.

Lerm, E., Engelbrecht, L. \& Du Toit, M., 2010. Malolactic fermentation: The ABC's of MLF. S. Afr. J. Enol. Vitic. 31(2), 186-212.

Lonvaud-Funel, A., 1995. Microbiology of the malolactic fermentation: Molecular aspects. FEMS Microbiol. Lett. 126(3), 209-214.

Lonvaud-Funel, A., 1999. Lactic acid bacteria in the quality improvement and depreciation of wine. Anton. Leeuw. 76, 317-331.

Massera, A., Soria, A., Catania, C., Krieger-Weber, S. \& Combina, M., 2009. Simultaneous inoculation of Malbec (Vitis vinifera) musts with yeast and bacteria: Effects on fermentation performance, sensory and sanitary attributes of wines. Food Technol. Biotech. 47(2), 192-201.

Miller, B.J., Franz, M.P., Cho, G.S. \& Du Toit, M., 2011. Expression of the malolactic enzyme gene (mle) from Lactobacillus plantarum under winemaking conditions. Curr. Microbiol. 62(6), 1682-1688.

Mtshali, P.S., Divol, B., Van Rensburg, P., \& Du Toit, M., 2010. Genetic screening of wine-related enzymes in Lactobacillus species isolated from South African wines. J. Appl. Microbiol. 108(4), 1389-1397.

Ribereau-Gayon, P., Dubordieu, D., Doneche, B. \& Lonvaud, A., 2006. Handbook of enology: The microbiology of wine and vinifications, vol. 1. John Wiley and Sons Ltd., New York.

Rosi, I., Fia, G. \& Canuti, V., 2003. Influence of different $\mathrm{pH}$ values and inoculation time on the growth and malolactic activity of a strain of Oenococcus oeni. Aust. J. Grape Wine R. 9, 194-199.

Vaillant, H., Formisyn, P. \& Gerbaux, V., 1995. Malolatic fermentation of wine: Study of the influence of some physico-chemical factors by experimental design assays. J. Appl. Bacteriol. 79(6), 640-650.

Wibowo, D., Eschenbruch, R., Davis, D.R., Fleet, G.H. \& Lee, T.H., 1985. Occurrence and growth of lactic acid bacteria in wine: A review. Am. J. Enol. Viticult. 36, 302-313. 